\title{
Wireless Communication Technologies for IoT in 5G: Vision, Applications, and Challenges
}

\author{
Quy Vu Khanh, ${ }^{1}$ Nam Vi Hoai, ${ }^{1}$ Linh Dao Manh, ${ }^{1}$ Anh Ngoc Le $\mathbb{D}^{2},{ }^{2}$ and Gwanggil Jeon $\mathbb{D}^{3}$ \\ ${ }^{1}$ Hung Yen University of Technology and Education, Hung Yen, Vietnam \\ ${ }^{2}$ Swinburne Vietnam, FPT University, Hanoi, Vietnam \\ ${ }^{3}$ Incheon National University, Incheon, Republic of Korea
}

Correspondence should be addressed to Anh Ngoc Le; anhngoc@epu.edu.vn and Gwanggil Jeon; gjeon@inu.ac.kr

Received 19 October 2021; Revised 4 November 2021; Accepted 4 January 2022; Published 7 February 2022

Academic Editor: Mohammad R Khosravi

Copyright (c) 2022 Quy Vu Khanh et al. This is an open access article distributed under the Creative Commons Attribution License, which permits unrestricted use, distribution, and reproduction in any medium, provided the original work is properly cited.

\begin{abstract}
Communication technologies are developing very rapidly and achieving many breakthrough results. The advent of 5th generation mobile communication networks, the so-called 5G, has become one of the most exciting and challenging topics in the wireless study area. The power of $5 \mathrm{G}$ enables it to connect to hundreds of billions of devices with extreme-high throughput and extreme-low latency. The $5 \mathrm{G}$ realizing a true digital society where everything can be connected via the Internet, well known as the Internet of Things (IoT). IoT is a technology of technologies where humans, devices, software, solutions, and platforms can connect based on the Internet. The formation of IoT technology leads to the birth of a series of applications and solutions serving humanity, such as smart cities, smart agriculture, smart retail, intelligent transportation systems, and IoT ecosystems. Although IoT is considered a revolution in the evolution of the Internet, it still faces a series of challenges such as saving energy, security, performance, and QoS support. In this study, we provide a vision of the Internet of Things that will be the main force driving the comprehensive digital revolution in the future. The communication technologies in the IoT system are discussed comprehensively and in detail. Furthermore, we also indicated indepth challenges of existing common communication technologies in IoT systems and future research directions of IoT. We hope the results of this work can provide a vital guide for future studies on communication technologies for IoT in $5 \mathrm{G}$.
\end{abstract}

\section{Introduction}

The development history of mobile communication systems demonstrated that aim to meet the requirements of humanity, the data rate of mobile communication is constantly being improved and achieved breakthrough results. Mobile generations have evolved through 5 periods, starting from $1 G$ to the current $5 G$ [1]. Network generations from $1 G$ to $3 \mathrm{G}$ have shown the continuous evolution of services and speeds. The 4G was proposed in the early 2000s. $4 \mathrm{G}$ was the first network generation entirely based on the IP packet switching method [2]. After about ten years of implementation, the former advantages of $4 \mathrm{G}$ have converted into disadvantages. Nowadays, $4 \mathrm{G}$ has access speed has become too low with high latency [3]. Humanity needs a solution to connect with data rates up to Gbps. The advent of the next- generation network called 5G in the early 2020s marks a comprehensive digital society. In particular, in 5G, a new concept is considered the Internet of Things (IoT) $[4,5]$. Io $\mathrm{T}$ is an integrated system of advanced technologies and solutions that allows devices, people, platforms, software, and solutions to be connected through the Internet $[6,7]$.

According to Cisco, more than 500 billion devices will be connected to the Internet by 2030 . These devices will be endogenously equipped with IoT modules that allow device-to-device (D2D) communications to each other, forming IoT networks [8]. IoT applications will be deployed in almost all humanity domains, including smart cities $[9$, 10], smart transportation $[11,12]$, smart agriculture [13, 14], and smart homes [15]. In [16], we presented a detailed survey of IoT applications for humanity. We illustrate several typical IoT applications as in Figure 1. 

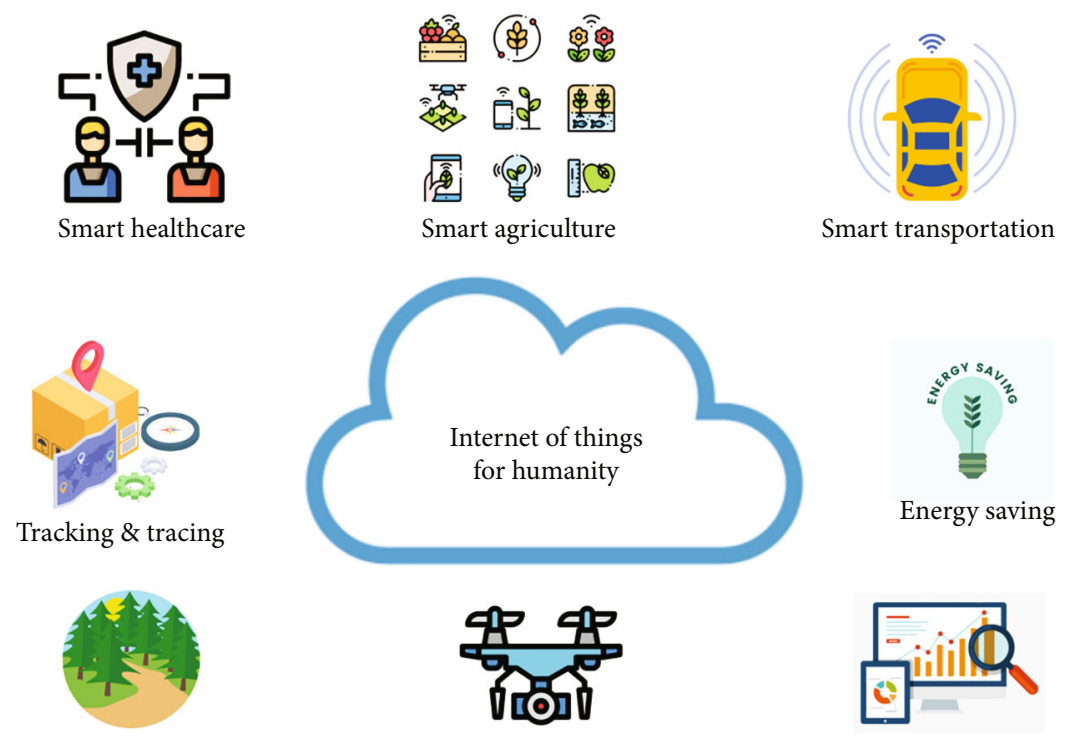

UAV communcation
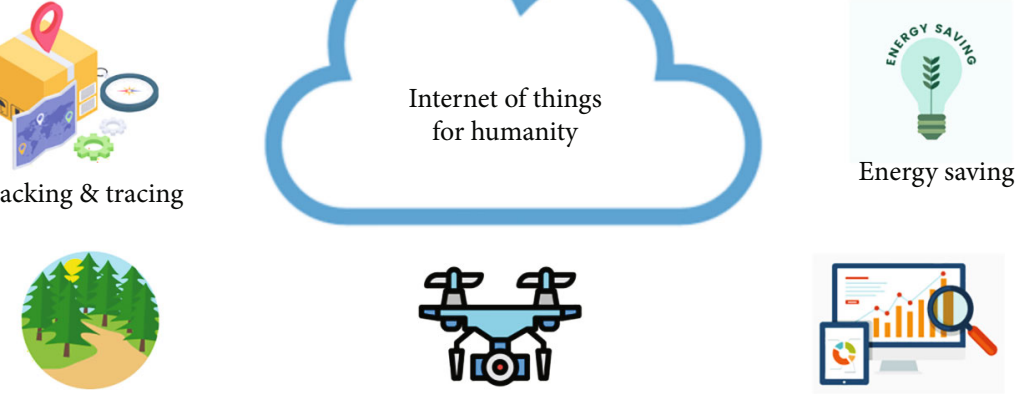

Monitoring forestry

FIgURE 1: An illustration of IoT applications for humanity.

However, the survey results also showed that IoT networks in $5 \mathrm{G}$ have a series of challenges such as performance improvement, support QoS, saving energy, privacy, and security $[17,18]$. Communication solutions including architecture, routing algorithm, protocol, and spectrum have been proposed to solve these problems. In this study, we conduct a comprehensive survey of communication technologies for IoT in 5G. The main contributions of this survey are listed as follows:

(i) The vision of the Internet of Things in 5G: architecture and research timeline

(ii) A comprehensive survey of the recent communication technologies for IoT in $5 \mathrm{G}$

(iii) The breakout technologies and solutions for IoT in $5 \mathrm{G}$

(iv) Challenges and attractive research topics in the future of communication for IoT

(v) The vision of the Internet of Things in 5G

The evolutionary history of network generations has proven that each generation is born to correct the weakness of previous generations and do some things that the previous generation could not do [19]. In the early 2020s, the Internet of Things concept was born simultaneously with the emergence of 5G [20]. Therefore, to define the vision of IoT in $5 \mathrm{G}$, we need to clarify the advent context of IoT in $5 \mathrm{G}$.

For the convenience of following the article, we have compiled the acronyms in Table 1.

1.1. Forming of IoT in 5G. The development history of mobile communication systems began in the early 1980s. During its development, mobile radio communication sys- tems always tend to integrate all systems. End-user devices are smarter more and more, lighter, save energy, support all types of data such as voice, video, and real-time multimedia applications. The data rate and bandwidth increase with costs decrease. The $1 \mathrm{G}-3 \mathrm{G}$ network generations are standardized and deployed widely worldwide, in references [1, 21]; so, we will not consider these issues to focus on presents the $4 \mathrm{G}$ network generation.

The $4^{\text {th }}$ mobile network generation $(4 \mathrm{G})$ is formed after $3 \mathrm{G}$ and before 5G. Besides the provided services of $3 \mathrm{G}$, it also provides added services such as broadband Internet access, IP phone (VoIP), video conferencing, online games, high-definition Internet TV, 3D TV, and cloud computing. The two technologies were standardized for $4 \mathrm{G}$ as Wimax and LTE [21]. One difference with previous generations, $4 \mathrm{G}$ unsupports the traditional circuit switching mechanism but relies entirely on IP protocol with the packet switching mechanism. Aim to speed up data transmission, spectrum modulation technologies of previous generations are replaced by OFDMA technology, combined with MIMO multipoint transceiver mechanism and smart antenna. [22]. As a result, the bit rate in $4 \mathrm{G}$ is significantly higher than in $3 \mathrm{G}$.

With many advantages mentioned above, $4 \mathrm{G}$ has become a pioneering technology and commercialized in many countries. In Vietnam, 4G was deployed in 2016 [23]. However, after many years of deployment, $4 \mathrm{G}$ has revealed the limitations of this network generation. According to Cisco, over 500 billion devices will be connected to the Internet in the future. This is beyond the provided capacity of 4G [24]. Moreover, the delay of $4 \mathrm{G}$ is too large for the real-time applications, approximately $10 \mathrm{~ms}$, and the data rate of $4 \mathrm{G}$ is relatively low, approx. 3 (Mbps). With the number of devices increasing hundreds of times today, $4 \mathrm{G}$ will consume a huge amount of energy.

The limitations of $4 \mathrm{G}$ were indicated that the advent of $5 \mathrm{G}$ is an inevitable trend. Humans need a new network 
TABle 1: Acronyms used in the survey and definations.

\begin{tabular}{|c|c|}
\hline Acronym & Definition \\
\hline 3GPP & 3rd generation partnership project \\
\hline $5 \mathrm{G}$ & 5th generation mobile networks \\
\hline AAC & Adaptive admission control \\
\hline AI & Artificial intelligence \\
\hline ANN & Artificial neural network \\
\hline $\mathrm{AR}$ & Augmented reality \\
\hline $\mathrm{D} 2 \mathrm{D}$ & Device-to-device \\
\hline $\mathrm{D} 2 \mathrm{D}$ & Device to device \\
\hline eNB & Evolved node B \\
\hline GPRS & General Packet Radio Service \\
\hline GSMA & Global System for Mobile Communications \\
\hline IIoT & Industrial Internet of Things \\
\hline IoT & Internet of Things \\
\hline IP & Internet protocol \\
\hline LoRa & Long range \\
\hline LoRaWAN & Long Range Wide-Area Network \\
\hline LPWANs & Low-Power Wide-Area Technologies \\
\hline LTE & Long-term evolution \\
\hline MIMO & Multiple in, multiple out \\
\hline NB-IoT & Narrowband IoT \\
\hline NFC & Near field communication \\
\hline OFDMA & Orthogonal frequency-division multiple access \\
\hline QoS & Quality of service \\
\hline RFID & Radio frequency identification \\
\hline SC-FDMA & Single-carrier FDMA \\
\hline SDN & Software-defined networking \\
\hline UAV & Unmanned aerial vehicle \\
\hline VoIP & Voice over internet protocol \\
\hline
\end{tabular}

generation that the data rate increases hundreds of times faster, but energy consumption reduces many times compared to $4 \mathrm{G}$. Some countries such as China, Korea, the United Kingdom, and the United States are currently pioneering in the studies and deployment of 5G. Although still not yet official standardized, GSMA and some organizations and suppliers such as Ericsson and Huawei have proposed the standard of 5G network generation [25] as follows:

Aim to achieve these goals, in Table 2, many breakout technologies and solutions need to be implemented synchronously. However, like previous generations, the improvement of the radio access layer has always been a significant challenge to meet the goals of 5G. In this study, we approach $5 \mathrm{G}$ from an Internet of Things perspective. The concept of IoT was first mentioned in $5 \mathrm{G}$. IoT is an advanced technology that allows things, machines, devices, solutions, and people to connect through the Internet. IoT is expected to become popular in all areas serving people, such as smart agriculture, smart transportation, smart cities, health, rescue and disaster recovery, retail, management house, and green energy. A very diverse survey of IoT applications is presented in [26].
TABLE 2: Main characteristics of 5G network generation.

\begin{tabular}{lc}
\hline Characteristics & Goal \\
\hline Mobile access speed & $1 \mathrm{Gbps}$ \\
Fixed access speed & $1-10 \mathrm{Gbps}$ \\
Data transmission delay & $1 \mathrm{~ms}$ \\
Reliability & $99.999 \%$ \\
Energy consumption & Reduce many times compared to 4G
\end{tabular}

1.2. The Architecture of IoT in 5G. IoT in the 5G framework consists of main four-layer architecture, as shown in Figure 2, and is related to data collection, processing, analysis, and sharing of information between equipment and communication networks.

(i) Thing layer: This layer includes physical systems such as actuators, devices, sensors, and communicates with the network layer

(ii) Network layer: The network layer consists of two sublayers: (1) low power wide area technologies (LPWANs) such as SigFox, LoRa, ZigBee, NB-IoT, and (2) backhaul-based connections of 5G. In this study, in order to focus on detailing communication solutions in IoT, communication technologies in the backhaul layer are not within the scope of this research

(iii) Middleware layer: this layer is considered the heart of the network. The IoT framework focuses on advanced technologies and solutions as fog computing, edge computing, cloud computing, AI vision, and big data analytics are deployed

(iv) Application layer: this layer presents IoT applications that are deployed in a series of domains as management factories and buildings, agriculture, traffic system, and IoT ecosystems. This layer integrates all solutions, technologies, and applications to interact with humans through the Internet connection

A specific illustration of this architecture is presented in Figure 3. The sensor devices of IoT applications interact with the IoT gateway based on low-power communication networks such as SigFox, LoRa, or NB-IoT. These IoT gateways collect information from IoT devices and then transmit it to the Cloud through the 5G backhaul communications. In the middleware layer, the collected data is processed and stored, combining autonomous decision-making systems or human controls to make under layer tasks.

1.3. Research Timeline IoT in 5G. Nowadays, study activities on different aspects of the Internet of Things in $5 \mathrm{G}$ are exciting in both academic research and industry. Some of the top mobile telecommunication corporations and excellent research labs perform studies and experiments to provide applications and solutions of IoT in 5G. 

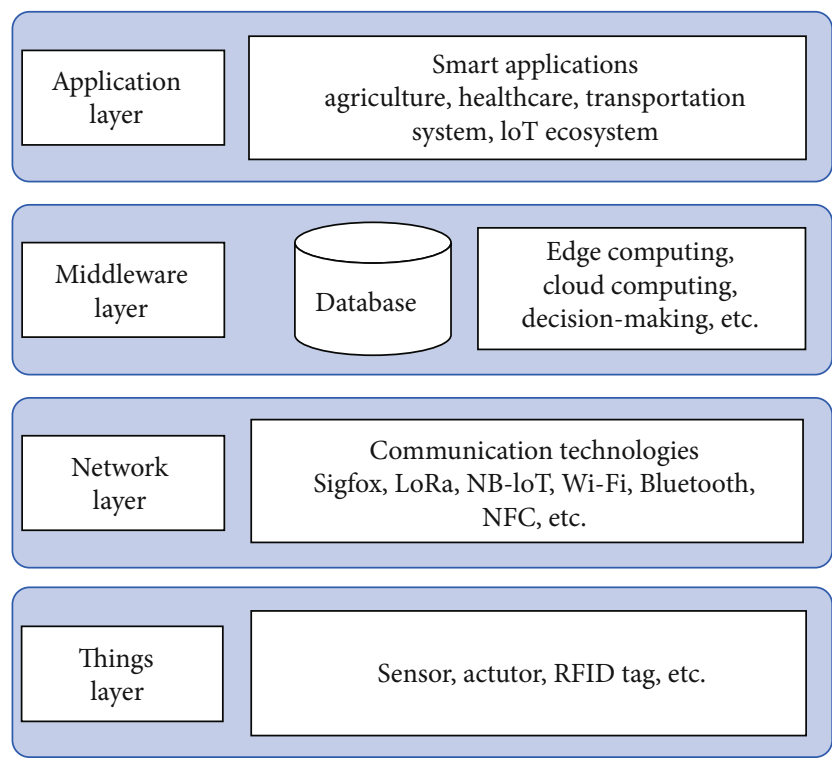

Figure 2: An illustration of the IoT in $5 \mathrm{G}$ architecture.

1.3.1. Intel. This corporation has pioneered in the IoT field. The company predicts that IoT devices will generate over $55 \%$ of global data by 2025 . In order to accelerate the application of IoT in various areas serving humanity, Intel is developing an IoT ecosystem at all layers of the IoT architecture with the key technologies and solutions [27] as follows:

(i) In thing layer, Intel providers unique performance scalability with four processor families for IoT applications. Besides, processors of Intel run a variety of operating systems such as Linux, Microsoft, and Google

(ii) In network layer, Intel supports many networking interfaces and protocols to provide the necessary connectivity. Besides, Intel also provides Gateway solutions for the IoT

(iii) In middleware layer, Intel server technology is extensively used in the network and cloud infrastructure. Moreover, Intel is focusing on three IoT computing projects, including edge computing, cloud computing, and $\mathrm{AI}$ and computer vision

(iv) In application layer, Intel provides foundations to support the IoT application in various other domains, such as Figure 4

1.3.2. Samsung. According to Samsung, the total number of IoT devices is expected to increase to 21.5 billion by 2025 . The number of devices also increases to 34.2 billion if it includes smartphones, laptops, and tablets. Furthermore, Samsung also forecasts that the global IoT market will archive around $\$ 1.600$ billion. Relying on the expectation that all devices will be connected to the Internet, Samsung has built IoT ecosystems in $5 \mathrm{G}$ to realize aspirations such as smart homes, smart cities, smart factories, healthcare, smart agriculture, and logistics [28]. Some of the recent developments in the field of IoT are as follows:

(i) In application layer, Samsung is providing IoT solutions that allow users to control home appliances. The Samsung electronic devices such as TVs, washing machines, and refrigerators can be controlled by remote based on a Samsung smartphone

(ii) In middleware layer, Samsung is implementing research projects related to optimal computing solutions, specifically edge computing, cud computing, and AI vision

(iii) In thing layer, products and devices designed for Samsung IoT platforms, including phones, tablets and wearables, digital signage, and automation solutions. In particular, Samsung designed the unique IoT modules, called Samsung ARTIK modules, which can be customized based on the size, ability, and capabilities of the Samsung products. Moreover, the Samsung ARTIK Smart IoT platform combines open-source modules and cloud services with an ecosystem of tools and partners that is motivation to drive the development of the IoT in 5G. Figure 5 is an illustration of the Samsung Artik 530 development kit

1.3.3. Ericsson. According to Ericsson, the expected IoT numbers of connections would increase over 3.5 times from about 1.7 billion in 2020 to approx 6 billion by 2026 . Erricson also forecasts there will have over 24 billion interconnected IoT devices Internet by 2050. Consequently, almost everything is around us as home appliances, vehicles, traffic lights, personal devices, learning devices, and health monitoring would be connected to the Internet. This will be a very exciting area both in academic and industrial research in the coming years. With the ambition to connect anything, anywhere, Ericsson is driving the growth of the IoT through its major contributions in the domain of realtime network performance and cloud computing solutions [29]. Some researches dedicated by Ericsson for IoT in 5G are as follows:

(i) In application layer, besides developing IoT solutions and applications for a wide range of fields such as healthcare and smart agriculture, Ericsson developed an IoT Accelerator Developer Portal to support the development of IoT solutions for the community of application developers worldwide, as presented in Figure 6

(ii) In network layer, Ericsson has focused on researching spectrum sharing solutions, exploiting mmWave, $\mathrm{THz}$ frequency bands, and intelligent communication solutions between devices

(iii) In middleware layer, Ericsson promotes research into architectures and solutions of cloud computing and edge computing 


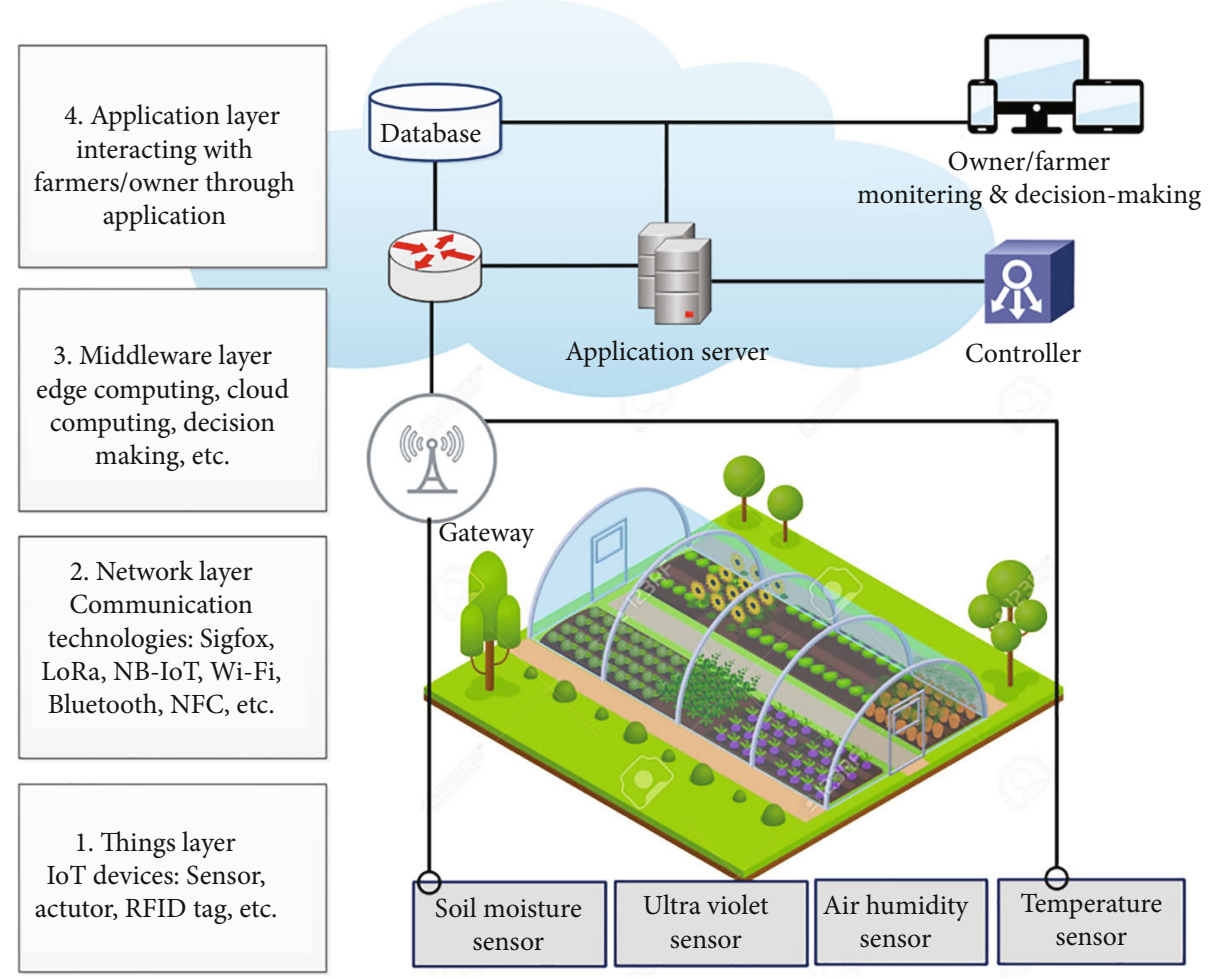

FIgURE 3: An illustration of practise IoT structure for smart agriculture area.

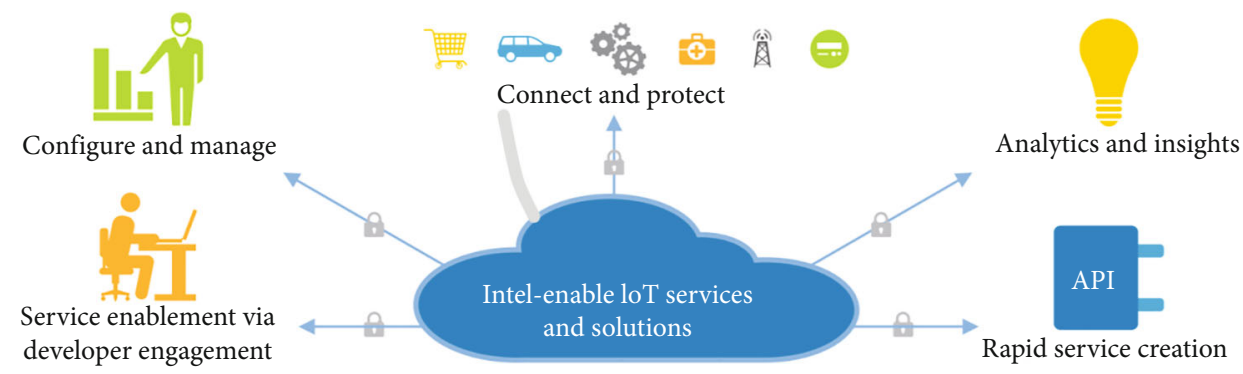

Figure 4: The foundation for connected IoT.

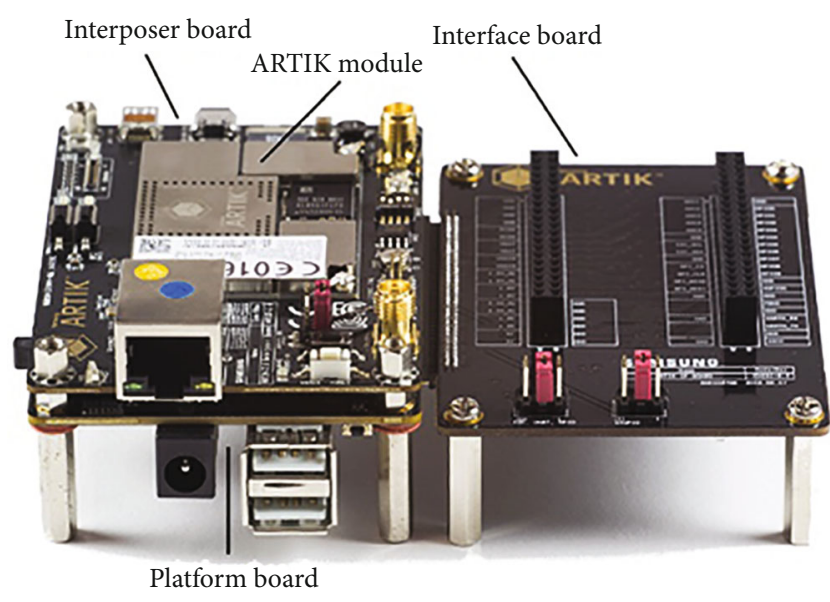

Figure 5: An illustration of the Samsung Artik 530 development kit.
1.3.4. Huawei. This corporation is a pioneering provider of communication solutions for IoT in $5 \mathrm{G}$ with a very diverse IoT ecosystem. Huawei has developed a Huawei IoT Connection Management Platform that aims to provide a full connection between people and things and fast integration for the vertical industry applications.

According to Huawei, promoting the development of IoT is based on five factors: (1) flexible deployment, (2) multiple connections, (3) intelligent management, (4) data security, and (5) open ecosystems. Reply to these factors, Huawei is driving the development of IoT through a series of significant contributions in all layers of IoT architecture, from the things layer to the application layer [30], as presented in Figure 7. Along with the achieved breakthrough study results by top telecommunication corporations, a series of research labs around the world are also driving the research process to find promising solutions for IoT in $5 \mathrm{G}$ aim enhance data rate, exploit spectrum more efficiently, extend communication distances, optimize energy consumption, 


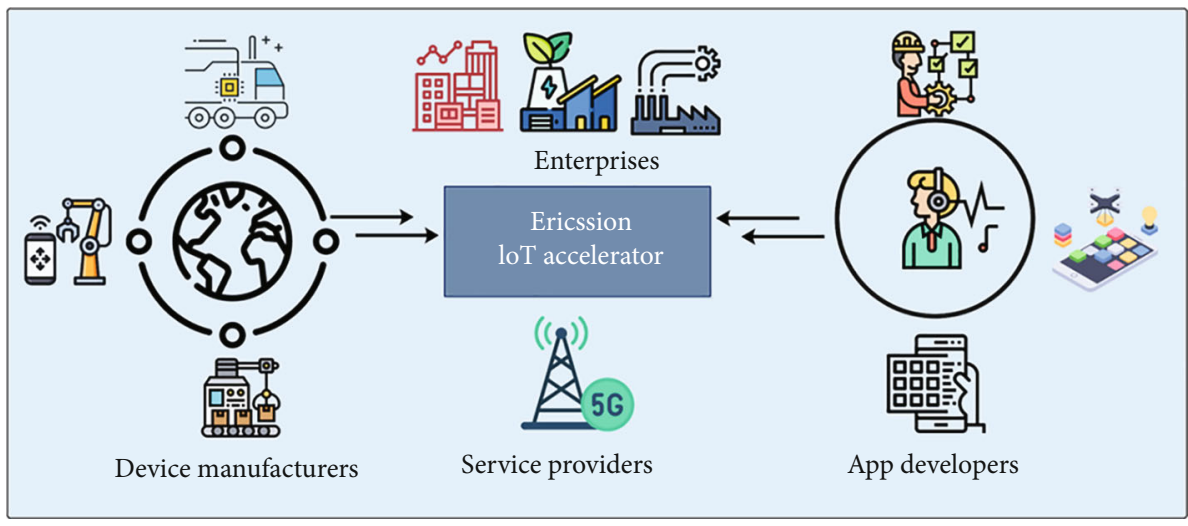

Figure 6: An illustration of IoT Accelerator Developer Portal.

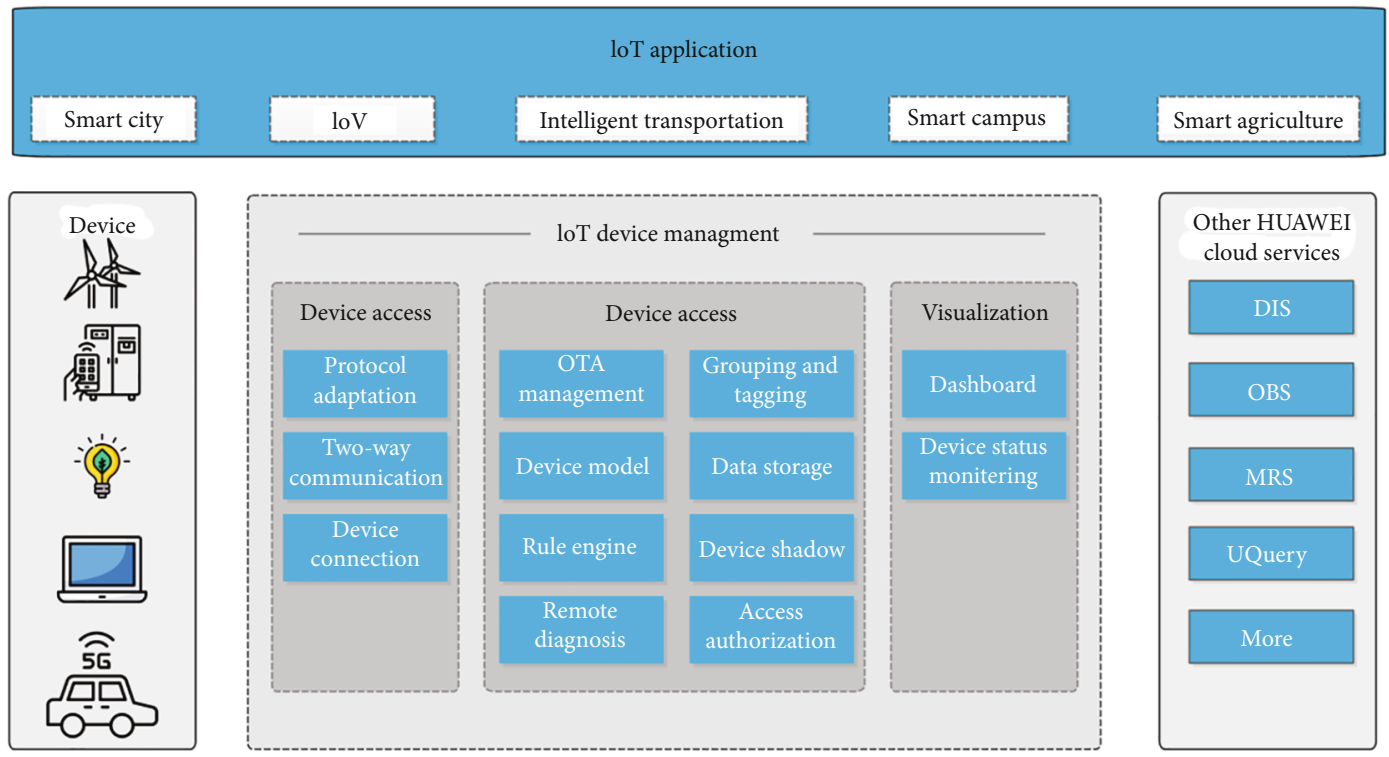

FIgURE 7: An illustration of solutions and products' architecture in the IoT domain of Huawei.

and extend scale networks up to hundreds of billions of Things. IoT in $5 \mathrm{G}$ could be the most revolutionary technology in the communication and information technology area. It could be applied in a series of different domains from popular applications in life such as payment utilities, smart retail, manage home appliances to expert apps such as selfdriving vehicles, monitoring traffic status, collision warning between vehicles and monitoring, and controlling green energy systems, smart cities management. In the agriculture area, IoT can also be applied in applications such as forestry management, farm management, monitoring forest fire, tracing, and tracking products. In the industrial area, actuators and robots with the support of AI technology can perform tasks day and night replace humans with extremely high productivity and accuracy. It realizes the dream of smart and green factories.

\section{Survey of Recent Communication Solutions}

Advances in the semiconductor, electronics, and automation industries are driving the development of communication solutions for IoT in 5G. These solutions are smarter, more reliable, robust, high data rate, and energy saving. As a result, various low-power communication technologies have been proposed for IoT in 5G, such as SigFox and LoRa. Survey results have demonstrated that low power technologies are suitable for IoT $5 \mathrm{G}$ networks due to their unique characteristics such as wide coverage, low power, high energy efficiency, and suitable data rate. In this section, we present the recently proposed communication solutions for IoT in $5 \mathrm{G}$. We divide these proposals into four categories based on technology. The detailed survey results are presented in the following subsections and are summarized in Table 3.

2.1. SigFox. SigFox technology was introduced in the 2010 s to connect low-power devices such as electricity meters and smartwatches, which need to be continuously operated on and have extremely low data rates. SigFox uses the industrial, scientific, and medical radio band, which uses $868 \mathrm{MHz}$ in Europe and $902 \mathrm{MHz}$ in the US with a channel bandwidth of $100 \mathrm{MHz}$. SigFox uses a wide-reaching signal that passes freely through solid objects, called ultra narrowband and 
TABLe 3: Some typical LPWAN communication technologies for IoT in 5G.

\begin{tabular}{|c|c|c|c|c|}
\hline Type & Transmission distance & Type of network & Frequency & Data rate \\
\hline $802.11 \mathrm{a} / \mathrm{b} / \mathrm{g} / \mathrm{n} / \mathrm{ac}$ & $100 \mathrm{~m}$ & WLAN & 2.4-5 GHz & 2-700 Mbps \\
\hline $802.11 \mathrm{ah}$ & $1000 \mathrm{~m}$ & WLAN & Sub-GHz & $78 \mathrm{Mbps}$ \\
\hline $802.11 \mathrm{p}$ & $1 \mathrm{~km}$ & WLAN & $5.9 \mathrm{GHz}$ & 3-27 Mbps \\
\hline 802.11 af & $1 \mathrm{~km}$ & WLAN & $54-790$ & 25-550 Mbps \\
\hline SigFox & $\begin{array}{l}\text { Rural: } 50 \mathrm{~km} \\
\text { Urban: } 10 \mathrm{~km}\end{array}$ & LPWA & Zwave & $100-600 \mathrm{bps}$ \\
\hline LoRaWAN & $20 \mathrm{~km}$ & LPWA & Sub-GHz & $0.3-100 \mathrm{Kbps}$ \\
\hline NB-IoT & $35 \mathrm{~km}$ & LPWA & Zwave & $250 \mathrm{Kbps}$ \\
\hline ZigBee & $1 \mathrm{~km}$ & LPWA & $2.4 \mathrm{GHz}$ & $250 \mathrm{Kbps}$ \\
\hline
\end{tabular}

requires low energy; so, it also is an LPWAN technology. It uses a one-hop star topology. SigFox is used to cover large areas and to reach underground objects. SigFox cells have a coverage range of about $30-50 \mathrm{~km}$ in rural areas and reduced to under $10 \mathrm{~km}$ in crowded areas. Overall, SigFox enables to provide a wide area network with low-power consumption. Nowadays, the SigFox IoT system has covered around 72 countries with over 1.3 billion of the world population. Several recent IoT applications are based on SigFox communication, as follows:

In [31], Joris et al. (2019) designed an autonomous SigFox sensor node capable collected data from an area of sensors, then transmitting data to the Cloud for smart agriculture applications. Aim to enhance the ability of this system, the sensor nodes are designed to use solar energy. Experimental data show that the system can transmit data every 5 minutes in cloudy conditions. In [32], Lavric et al. (2019) analyzed the responsiveness of SigFox under different scale and density conditions of sensors for IoT networks. The figures indicated that the maximum number of sensors that can transmit data at the same time is approximately 100. The results indicated that, as the number of sensors increases above 100, the network performance could be decreased. Moreover, this study also proposes solutions to improve performance, large-scale, and high-density of sensors in SigFox IoT networks.

In [33], Mikhaylov et al. (2019) evaluated the performance of SigFox communication technology in the real world. Specifically, they deployed a SigFox-based communications network at 311 different locations in Brno city, Czech Republic. Then, they conduct tests to evaluate the performance and characteristics of the radio channel. The experimental results show that the packet delivery ratio achieved over $94 \%$ in the urban environment in the real world.

2.2. LoRa. The LoRa is emerging as one of the most promising low-power wide-area (LPWA) communication technologies. It enables the energy-constraint devices distributed over wide-scale areas to establish connectivity at an affordable cost. The LoRa uses a low-power wide-area network modulation technique and unlicensed frequency bands like $433 \mathrm{MHz}, 868 \mathrm{MHz}$ (Europe), $915 \mathrm{MHz}$ (Australia and North America), and $923 \mathrm{MHz}$ (Asia). LoRa enables long range transmissions with low power consumption. The LoRa technology covers the physical layer, while other technologies and protocols such as LoRaWAN (Long Range Wide-Area Network) cover the network layer. Depending upon the spreading factor, it can achieve data rates between $0.3 \mathrm{kbps}$ and $27 \mathrm{kbps}$. However, how to implement a flexible LoRa network with an effective cost is still an open challenge.

In [34], Zhou et al. (2019) designed and introduced an open LoRa system for IoT networks. Contributions of this work include (1) design and hardware implementation of a LoRa gateway, (2) use LoRa open-source codes on GitHub, and (3) improve server LoRa through the uses of the messages system for the interaction between modules to guarantee scalability and flexibility. The experimental results have shown that the proposed system has improved the performance of the LoRa network compared to the traditional LoRa network.

In [35], Lee et al. (2018) designed and evaluated the performance of a LoRa mesh network to examine the applicability of LoRa networks for urban scenarios. This work installed 19 mesh LoRa devices in range [800×600] $\mathrm{m}$ on a university campus and installed a gateway that collected data at 1-min intervals. The experimental results showed that the proposed LoRa system has an average packet delivery ratio of $88.49 \%$, whereas the star LoRa topology only achieved $58.7 \%$ under the same conditions.

The LoRa is one of the most successful technologies of the LPWAN (Low-Power Wide-Area Network) family. It enables robust long-distance low power communications and is proven to be effective in the Internet of Things (IoT) applications. The LoRa is also promising for Industrial IoT scenarios. However, a limitation of LoRa does not offer support to real-time data flows. To solve this problem, In [36], Leonardi et al. (2019) proposed a new medium access strategy for LoRa, called RT-LoRa, which aim to support real-time LoRa-based IoT applications. The simulation results demonstrated that RT-LoRa could support real-time flows for IoT applications.

2.3. Wi-Fi. Wi-Fi is a known-well family of wireless communication technologies based on the IEEE 802.11 family of standards. It is commonly used for local area networks of devices and Internet access within 100 (m). It operates in the $2.4-5 \mathrm{GHz}$ frequency band. Wi-Fi is suitable for shortrange communication; so, it is a feasible communication solution for IoT networks. 
In [37], Pokhrel et al. (2020) proposed a queue management solution for the home IoT access points based on Wi-Fi. The focus of this work proposal adaptive admission control mechanism at the Wi-Fi access point aims to reduce the response time of the access point. The experiment results demonstrated that the proposed system is more stable than traditional home IoT systems based on Wi-Fi. In [38], Sheth et al. (2019) proposed a saving energy communication solution based on WIOTAP for IoT systems based on Wi-Fi. The focus of this work uses an intelligent $\mathrm{Wi}$-Fi access point. Then, it presents a downlink packet scheduling mechanism to reduce downlink channel access contention and queuing delay of regular stations in IoT systems. The results demonstrated that the proposed system improved over $38 \%$ of energy consumption and over $41 \%$ of the delay compared to traditional solutions.

Real-time locating and tracking are the most critical problems of IoT applications. GPS-based positioning applications are well known for outdoor environments. However, it is not feasible for indoor scenarios. In [39], Ruo et al. (2019) proposed an IoT solution to tracking and locating indoor based on Wi-Fi signals for indoor environments. The focus of this work uses a message type that is built-in the 802.11-REVmc2 Wi-Fi standard. Then, through measurements of the roundtrip time and signal strength to improve the accuracy and ability of the positioning system. Experiment results demonstrated that the proposed system enhanced the performance and achieved an average positioning accuracy of $1.435 \mathrm{~m}$ with an update time of every $0.19 \mathrm{~s}$ for indoor scenarios.

2.4. ZigBee. ZigBee is a communication technology that uses the IEEE.802.15.4 standard and operates in the industrial, scientific, and medical radio frequency bands. It is a low-power wide-area communication solution for IoT networks. ZigBee technology in IoT networks has advantages compared to other communication technologies because of its simplicity, flexibility, and low cost. The transmission distance of ZigBee is about $100 \mathrm{~m}$, with a data rate that is about $250 \mathrm{kbps}$, depending on power output and environmental features. ZigBee is typically used in extreme-low data rate networks, short-range, and long-lasting battery life such as home automation, medical device data collection, and industrial equipment control.

In [40], Pirayesh et al. (2021) proposed a ZigBee receiver based on MIMO against jamming attacks for IoT networks. This work designed a prototype of the ZigBee receiver based on MIMO technology and a learning mechanism to mitigate the unknown interference. The experiment results demonstrated that the proposed system could provide an average of over $26.7 \mathrm{~dB}$ jamming mitigation capability compared to the traditional ZigBee receiver.

In [41], Farha et al. (2021) introduced a new security schema based on a timestamp against replay attacks for ZigBee networks. This solution improves energy consumption significantly. Besides, to enhance feasibility, this solution uses powered devices to provide energy for powerconstrained devices with the current timestamp. The proposal is designed to be suitable for all ZigBee networks. The experiment results indicated that the proposed solution improves significantly against ability reply attacks in the ZigBee-based IoT networks.

In [42], Ali et al. (2019) designed the smart sensors that combined two communication modules include ZigBee and LoRa, to measure temperature and humidity factors for IoT applications. The collected data from sensors are sent to the central receiver unit by using the ZigBee or LoRa transceiver modules. The choice of the communication module can be controlled remote or based on the Cloud. The practical design and experiment figures indicated the benefits of the low-power, long range communication solutions for IoT applications.

2.5. Narrowband Internet of Things. Narrowband Internet of Things (NB-IoT) is a new LPWAN radio technology developed by 3GPP to support massive connections, wide-area coverage, ultra-low power consumption, and low cost for IoT in 5G. NB-IoT is a promising emerging communication technology for IoT in 5G. NB-IoT focuses specifically on indoor coverage, low cost, long battery life, and high connection density. It uses the bandwidth to narrow-band $200 \mathrm{kHz}$ and OFDM modulation for downlink communication and SC-FDMA for uplink communications.

In [43], Chen et al. (2020) designed a prototype NB-IoT network based on open source for IoT in $5 \mathrm{G}$ applications. The open-source NB-IoT results from cooperation between three providers, including EURECOM, B-COM, and NTUST, based on the open-source eNB of LTE technology. This work presents a method to use the existing commercial NB-IoT module to transmit the collected data from sensors to the Internet via the open-source NB-IoT network.

In [44], Chen et al. (2019) evaluated the performance and improved NB-IoT protocol for IoT networks in 5G. The focus of this work includes the following: (1) use the stochastic network to analyze the delay metric in the NB-IoT system and (2) improve NB-IoT protocol through the improvement of the $k$ -means algorithm to cluster NB-IoT devices and perform a scheduling strategy based on the priority. The experiment results indicated that the proposed uplink traffic scheduling schema enhanced performance compared to existing uplink traffic scheduling schemas.

In [45], Kanj et al. (2020) introduced a method to design the physical layer of the NB-IoT device. The focus of this work presents the characteristics and the scheduling of downlink and uplink physical channels at the NB-IoT base station and end-user device to help readers without having to read all the 3GPP specifications.

\section{Discussions, Challenges, and Open Issues}

In this study, we have highlighted the revolutionary contributions of IoT in $5 \mathrm{G}$ in a wide range of fields to serve humanity. Low power communication technologies will play an essential role in supporting and driving IoT applications more public. The survey results have indicated that many applications have been presented in Table 4 . The proposals are applied in a variety of domains such as environment, city, home, building, factory, and agriculture.

Communication technologies such as ZigBee, SigFox, LoRa, and NB-IoT have advantages such as low energy 
TABLE 4: Statistics of recently proposed IoT applications based on communication technologies in 5G.

\begin{tabular}{|c|c|c|c|}
\hline Ref. no & Technology & Application domain & Case study: Key focus \\
\hline$[31]$ & SigFox & Agriculture & $\begin{array}{c}\text { This proposal aims to design an autonomous IoT sensor to collect data in smart } \\
\text { agriculture based on the solar energy system. }\end{array}$ \\
\hline [32] & SigFox & $\begin{array}{l}\text { City, industry, building, } \\
\text { home, traffic }\end{array}$ & $\begin{array}{l}\text { This research evaluates the performance of the SigFox communication protocol under } \\
\text { different scale and density conditions. }\end{array}$ \\
\hline [33] & SigFox & City, environment & $\begin{array}{l}\text { This research deployed a network system in the real world at } 311 \text { locations of Brno city } \\
\text { to measure the real performance of SigFox. }\end{array}$ \\
\hline$[34]$ & LoRa & $\begin{array}{l}\text { City, environment, } \\
\text { healthcare, agriculture }\end{array}$ & $\begin{array}{c}\text { This research designs and deployment a LoRa network for performance improvement, } \\
\text { flexible, and reduced cost. }\end{array}$ \\
\hline [35] & LoRa & Building & $\begin{array}{l}\text { This research real deployed a LoRa mesh system on a university campus to consider the } \\
\text { real system performance. }\end{array}$ \\
\hline [36] & LoRa & Industry & $\begin{array}{l}\text { This research introduced a medium access strategy for LoRA, called RT-LoRa, to reduce } \\
\text { service response time for real-time IIoT applications based on LoRa communication } \\
\text { protocol. }\end{array}$ \\
\hline [37] & Wi-Fi & Home & $\begin{array}{l}\text { This research introduced the AAC mechanism at the Wi-Fi access point to reduce } \\
\text { service response time for home Wi-Fi IoT applications. }\end{array}$ \\
\hline [38] & $\mathrm{Wi}-\mathrm{Fi}$ & Home & $\begin{array}{c}\text { This research introduced the Wi-Fi IoT access point (Wiotap) to address saving energy } \\
\text { and reducing delay for home IoT applications. }\end{array}$ \\
\hline [39] & Wi-Fi & Home & $\begin{array}{l}\text { This research introduced a tracking and location IoT solution based on Wi-Fi to } \\
\text { improve accuracy and reduce delay indoor environments. }\end{array}$ \\
\hline$[40]$ & ZigBee & Security IoT networks & $\begin{array}{l}\text { This research designs a ZigBee receiver against jamming attacks for IoT networks based } \\
\text { on MIMO technology. }\end{array}$ \\
\hline [41] & ZigBee & Security IoT networks & $\begin{array}{l}\text { This research proposes a new security schema based on a timestamp against ability reply } \\
\text { attacks in the ZigBee-based IoT networks. }\end{array}$ \\
\hline [42] & ZigBee & Environment & $\begin{array}{l}\text { This research designs an IoT sensor that combines two communication modules, ZigBee } \\
\text { and LoRa, to improve energy consumption and performance. }\end{array}$ \\
\hline [43] & NB-IoT & IoT ecosystems & $\begin{array}{c}\text { This research designs a prototype NB-IoT network based on open source for IoT in } 5 \mathrm{G} \\
\text { applications. }\end{array}$ \\
\hline$[44]$ & NB-IoT & IoT ecosystems & $\begin{array}{l}\text { This research introduced a scheduling schema to improve the NB-IoT protocol to } \\
\text { enhance performance. }\end{array}$ \\
\hline [45] & NB-IoT & IoT ecosystems & $\begin{array}{l}\text { This research presents how to design the physical layer of the NB-IoT device according } \\
\text { to } 3 \mathrm{GPP} \text {. }\end{array}$ \\
\hline
\end{tabular}

consumption, large-coverage, use of unlicensed frequency bands, and suitable for the characteristics of IoT networks and increasingly popular applied in IoT applications. Communication solutions of IoT in $5 \mathrm{G}$ aim to provide connectivity for IoT applications. With hundreds of billions of IoT devices connected to the network, these technologies face several significant challenges. In our opinion, the two crucial issues are the security-aware and energy efficiency. Then, we present the challenges of communication technologies for IoT applications in $5 \mathrm{G}$ and indicate possible research directions.

3.1. Privacy and Security. The Internet of Things development forms a truly open world, where everything is connected to the Internet. Consequently, objects are easily vulnerable to attacks from the Internet. Therefore, according to Roukounaki et al. (2019) [46], privacy and security are the most critical factors to promote the development of IoT applications to become popular. In IoT applications, attacks can be performed in multiple layers, specifically as

(i) Security for IoT devices: the IoT devices with low computing capability and massive numbers are unsuitable for setting up robust security algorithms.
Consequently, attacks focus on exploiting the vulnerabilities of IoT devices

(ii) Security for gateway devices: the gateway devices play an important role in communication between things layer devices and upper layers. As a result, it is the heart of IoT applications. Denial of service attacks or data spoofing always focuses on the gateway of IoT applications

(iii) Security for devices at the edge: recently proposed solutions use edge computing technology to reduce service response times for real-time IoT applications. Consequently, the security of edge computing servers is one of the major challenges

(iv) Security for cloud servers: with the huge amount of data is provided by IoT devices, cloud infrastructure will be a possible solution in storing and processing big data. Consequently, the security of cloud servers will be one of the significant challenges

In [47], Zhou et al. (2021) presented a survey comprehensive of security logic bugs in IoT devices, platforms, 
and systems in all layers. In [48], Lins et al. (2021) presented a complete picture of potential threats as well as solutions aimed to mitigate attacks on IoT gateways. In [49], Wang et al. (2018) presented potential risks at the application layer, including data collection, storage, and data processing in the cloud of cloud-based IoT systems. Attacks into cloud servers to gain control or execute tasks to affect autonomous devices in smart factories and farms. In [50], Hassija et al. (2019) presented a diverse survey of attacks and security threats and proposed several architectural solutions to mitigate attacks on IoT systems.

In our opinion, security is one of the most critical problems of communication solutions in the IoT 5G network. This issue will continue to be a research topic timely and attract both academic and industry researchers in the future.

3.2. Energy Efficiency. Assuming that when IoT applications in $5 \mathrm{G}$ become popular, tens of billions of IoT devices will operate and transmit data continuously day and night. As a result, it will consume a huge amount of energy while energy resource is exhausted day by day. This is not feasible. Therefore, energy-efficient communication solutions are a real challenge.

In [51], Popli et al. (2019) presented a comprehensive survey of energy-saving solutions for IoT systems based on NB-IoT technology. The survey concluded that NB-IoT technology would be an essential technology to realize green IoT networks in the future. In [52], Ding et al. (2019) presented an optimal scheduling solution based on the multiobjective fuzzy algorithm to save energy for IoT networks. In [53], Al-Kadhim et al. (2019) presented a reliable and saving energy data transmission solution for cloud-based IoT systems. The figures demonstrate that the proposed solution reduced energy consumption by $57 \%$ and improved reliability by $60 \%$ compared to the traditional solution.

In our opinion, energy efficiency can be considered based on some of the solutions as follows:

(i) Communication technology-based: integration of smart, flexibly, and low-power communication technologies such as NB-IoT and ZigBee. In [17], the authors presented a survey of the energy harvesting communication technology for autonomously power IoT devices. This technology promises green energy in the future

(ii) Trade-off based: In reality, performance and energysaving have an antagonistic relationship. Therefore, a smart, flexible trade-off solution should be considered. In [54], Couso et al. (2018) proposed a trade-off solution for inverters to balance energy saving and performance for IoT-based smart grid applications

(iii) Cloud-based IoT networks: cloud will continue to be the backhaul infrastructure for IoT applications due to its robust storage, computing, and processing ability. However, cloud services have a high response time due to the edge computing solutions that are proposed. Consequently, an intelligent offload schema to optimal resource allocation between cloud and edge servers should be considered. In [55], Aljanabi et al. (2021) proposed a hybrid fogcloud offloading schema to optimal performance and energy for IoT applications

\section{Conclusion}

In this study, we introduced the vision, architecture, wireless communication technologies, and research timelines of IoT in 5G. Based on the analysis of the core components for IoT in $5 \mathrm{G}$, we conducted a short survey of low power communication technologies for IoT in $5 \mathrm{G}$. The survey results showed that the Internet of Things would be the future of humanities, where all things such as software, systems, and people are connected through the Internet. The advent of IoT in $5 \mathrm{G}$ led to the formation of a series of applications serving humanity, such as smart homes, smart cities, smart agriculture, smart factories, green energy, and IoT systems. Besides, we have provided a full picture of promising communication technologies for IoT in 5G such as SigFox, LoRa, Wi-Fi, and LoRaWAN. These solutions are suitable for the operating characteristics of IoT networks such as large coverage areas, high energy efficiency, and low energy consumption level, which support a large number of IoT devices.

Moreover, the survey results also point out some challenges of communication technologies for IoT in $5 \mathrm{G}$, including (1) privacy and security and (2) saving energy. In our opinion, the security and saving energy problems of communication technologies will continue to be exciting research topics in the future and receive attention from both academic research and industry. We hope that this study will play an important role as a guide for future research on communication technologies for IoT applications in $5 \mathrm{G}$.

\section{Data Availability}

No data were used to support this study.

\section{Conflicts of Interest}

The authors declare that they have no conflicts of interest.

\section{References}

[1] J. A. del Peral-Rosado, R. Raulefs, J. A. López-Salcedo, and G. Seco-Granados, "Survey of cellular mobile radio localization methods: from $1 \mathrm{G}$ to 5G," IEEE Communications Surveys \& Tutorials, vol. 20, no. 2, pp. 1124-1148, 2018.

[2] M. Agiwal, A. Roy, and N. Saxena, "Next generation 5G wireless networks: a comprehensive survey," IEEE Communications Surveys \& Tutorials, vol. 18, no. 3, pp. 1617-1655, 2016.

[3] M. Agiwal, H. Kwon, S. Park, and H. Jin, "A survey on 4G-5G dual connectivity: road to $5 \mathrm{G}$ implementation," IEEE Access, vol. 9, pp. 16193-16210, 2021.

[4] R. Khan, P. Kumar, D. N. K. Jayakody, and M. Liyanage, "A survey on security and privacy of $5 \mathrm{G}$ technologies: potential solutions, recent advancements, and future directions," IEEE Communications Surveys \& Tutorials, vol. 22, no. 1, pp. 196248,2020 
[5] L. Chettri and R. Bera, "A comprehensive survey on Internet of Things (IoT) toward 5G wireless systems," IEEE Internet of Things Journal, vol. 7, no. 1, pp. 16-32, 2020.

[6] S. Sinche, D. Raposo, N. Armando et al., "A survey of IoT management protocols and frameworks," IEEE Communications Surveys \& Tutorials, vol. 22, no. 2, pp. 1168-1190, 2020.

[7] M. Stoyanova, Y. Nikoloudakis, S. Panagiotakis, E. Pallis, and E. K. Markakis, "A survey on the internet of things (IoT) forensics: challenges, approaches, and open issues," IEEE Communications Surveys \& Tutorials, vol. 22, no. 2, pp. 1191-1221, 2020.

[8] https://www.cisco.com/c/en/us/products/collateral/se/ internet-of-things/at-a-glance-c45-731471.html.

[9] J. An, F. le Gall, J. Kim et al., "Toward global IoT-enabled smart cities interworking using adaptive semantic adapter," IEEE Internet of Things Journal, vol. 6, no. 3, pp. 5753-5765, 2019.

[10] F. Cirillo, D. Gómez, L. Diez, I. Elicegui Maestro, T. B. J. Gilbert, and R. Akhavan, "Smart city IoT services creation through large-scale collaboration," IEEE Internet of Things Journal, vol. 7, no. 6, pp. 5267-5275, 2020.

[11] A. J. V. Neto, Z. Zhao, J. J. P. C. Rodrigues, H. B. Camboim, and T. Braun, "Fog-based crime-assistance in smart IoT transportation system," IEEE Access, vol. 6, pp. 11101-11111, 2018.

[12] F. Zhu, Y. Lv, Y. Chen, X. Wang, G. Xiong, and F. -Y. Wang, "Parallel transportation systems: toward IoT-enabled smart urban traffic control and management," IEEE Transactions on Intelligent Transportation Systems, vol. 21, no. 10, pp. 4063-4071, 2020.

[13] O. Friha, M. A. Ferrag, L. Shu, L. Maglaras, and X. Wang, "Internet of Things for the future of smart agriculture: a comprehensive survey of emerging technologies," IEEE/CAA Journal of Automatica Sinica, vol. 8, no. 4, pp. 718-752, 2021.

[14] M. S. Farooq, S. Riaz, A. Abid, K. Abid, and M. A. Naeem, "A survey on the role of IoT in agriculture for the implementation of smart farming," IEEE Access, vol. 7, pp. 156237-156271, 2019.

[15] H. Uddin, M. Gibson, G. A. Safdar et al., "IoT for 5G/B5G applications in smart homes, smart cities, wearables and connected cars," in 2019 IEEE 24th International Workshop on Computer Aided Modeling and Design of Communication Links and Networks (CAMAD), pp. 1-5, Limassol, Cyprus, 2019.

[16] A. Kirimtat, O. Krejcar, A. Kertesz, and M. F. Tasgetiren, "Future trends and current state of smart city concepts: a survey," IEEE Access, vol. 8, pp. 86448-86467, 2020.

[17] D. Ma, G. Lan, M. Hassan, W. Hu, and S. K. Das, "Sensing, computing, and communications for energy harvesting IoTs: a survey," IEEE Communications Surveys \& Tutorials, vol. 22, no. 2, pp. 1222-1250, 2020.

[18] F. Hussain, R. Hussain, S. A. Hassan, and E. Hossain, "Machine learning in IoT security: current solutions and future challenges," IEEE Communications Surveys \& Tutorials, vol. 22, no. 3, pp. 1686-1721, 2020.

[19] M. H. Alsharif and R. Nordin, "Evolution towards fifth generation $(5 \mathrm{G})$ wireless networks: current trends and challenges in the deployment of millimetre wave, massive MIMO, and small cells," Telecommunication Systems, vol. 64, no. 4, pp. 617-637, 2017.

[20] K. Shafique, B. A. Khawaja, F. Sabir, S. Qazi, and M. Mustaqim, "Internet of things (IoT) for next-generation smart systems: a review of current challenges, future trends and prospects for emerging 5G-IoT scenarios," IEEE Access, vol. 8, pp. 2302223040, 2020.

[21] https://www.ericsson.com/en/blog/2020/4/impact-of-mobilenetwork-generations-on-business/.

[22] T. Mumtaz, S. Muhammad, M. I. Aslam, and N. Mohammad, "Dual connectivity-based mobility management and data Split mechanism in 4G/5G cellular networks," IEEE Access, vol. 8, pp. 86495-86509, 2020.

[23] S. Won and S. W. Choi, "Three decades of 3GPP target cell search through 3G, 4G, and 5G," IEEE Access, vol. 8, pp. 116914-116960, 2020.

[24] H. Beyranvand, M. Lévesque, M. Maier, J. A. Salehi, C. Verikoukis, and D. Tipper, "Toward 5G: FiWi enhanced LTE-A HetNets with reliable low-latency fiber backhaul sharing and Wi-Fi offloading," IEEE/ACM Transactions on Networking, vol. 25, no. 2, pp. 690-707, 2017.

[25] K. Samdanis and T. Taleb, "The road beyond 5G: a vision and insight of the key technologies," IEEE Network, vol. 34, no. 2, pp. 135-141, 2020.

[26] J. Ding, M. Nemati, C. Ranaweera, and J. Choi, "IoT connectivity technologies and applications: a survey," IEEE Access, vol. 8, pp. 67646-67673, 2020.

[27] "Intel," https://www.intel.com/.

[28] "Samsung," https://www.samsung.com/ph/.

[29] "IoT Platform," https://www.ericsson.com/en.

[30] "Huawei," https://www.huaweicloud.com/intl/en-us/.

[31] L. Joris, F. Dupont, P. Laurent, P. Bellier, S. Stoukatch, and J. M. Redoute, "An autonomous Sigfox wireless sensor node for environmental monitoring," IEEE Sensors Letters, vol. 3, no. 7, pp. 1-4, 2019.

[32] A. Lavric, A. I. Petrariu, and V. Popa, "Long range SigFox communication protocol scalability analysis under large-scale, high-density conditions," IEEE Access, vol. 7, pp. 3581635825, 2019.

[33] K. Mikhaylov, M. Stusek, P. Masek et al., "Communication performance of a real-life wide-area low-power network based on Sigfox technology," in ICC 2020-2020 IEEE International Conference on Communications (ICC), pp. 1-6, Dublin, Ireland, 2020.

[34] Q. Zhou, K. Zheng, L. Hou, J. Xing, and R. Xu, "Design and implementation of open LoRa for IoT," IEEE Access, vol. 7, pp. 100649-100657, 2019.

[35] H. Lee and K. Ke, "Monitoring of large-area IoT sensors using a LoRa wireless mesh network system: design and evaluation," IEEE Transactions on Instrumentation and Measurement, vol. 67, no. 9, pp. 2177-2187, 2018.

[36] L. Leonardi, F. Battaglia, and L. Lo Bello, "RT-LoRa: a medium access strategy to support real-time flows over LoRa-based networks for industrial IoT applications," IEEE Internet of Things Journal, vol. 6, no. 6, pp. 10812-10823, 2019.

[37] S. R. Pokhrel, H. L. Vu, and A. L. Cricenti, "Adaptive admission control for IoT applications in home Wi-Fi networks," IEEE Transactions on Mobile Computing, vol. 19, no. 12, pp. 2731-2742, 2020.

[38] J. Sheth and B. Dezfouli, "Enhancing the energy-efficiency and timeliness of IoT communication in Wi-Fi networks," IEEE Internet of Things Journal, vol. 6, no. 5, pp. 9085-9097, 2019.

[39] G. Guo, R. Chen, F. Ye, X. Peng, Z. Liu, and Y. Pan, "Indoor smartphone localization: a hybrid Wi-Fi RTT-RSS ranging approach," IEEE Access, vol. 7, pp. 176767-176781, 2019. 
[40] H. Pirayesh, P. Kheirkhah Sangdeh, and H. Zeng, "Securing ZigBee communications against constant jamming attack using neural network," IEEE Internet of Things Journal, vol. 8, no. 6, pp. 4957-4968, 2021.

[41] F. Farha, H. Ning, S. Yang, J. Xu, W. Zhang, and K. K. R. Choo, "Timestamp scheme to mitigate replay attacks in secure ZigBee networks," IEEE Transactions on Mobile Computing, vol. 21, no. 1, pp. 342-351, 2022.

[42] S. Z. Ali, S. K. Partal, and H. P. Partal, "ZigBee and LoRa based wireless sensors for smart environment and IoT applications," in 2019 1st global power, Energy and Communication Conference (GPECOM), pp. 19-23, Nevsehir, Turkey, 2019.

[43] C. Chen, R.-G. Cheng, C.-Y. Ho, M. Kanj, B. Mongazon-Cazavet, and N. Nikaein, "Prototyping of open source NB-IoT network," in GLOBECOM 2020-2020 IEEE Global Communications Conference, pp. 1-5, Taipei, Taiwan, 2020.

[44] X. Chen, Z. Li, Y. Chen, and X. Wang, "Performance analysis and uplink scheduling for QoS-aware NB-IoT networks in mobile computing," IEEE Access, vol. 7, pp. 44404-44415, 2019.

[45] M. Kanj, V. Savaux, and M. Le Guen, "A tutorial on NB-IoT physical layer design," IEEE Communications Surveys \& Tutorials, vol. 22, no. 4, pp. 2408-2446, 2020.

[46] A. Roukounaki, S. Efremidis, J. Soldatos, J. Neises, T. Walloschke, and N. Kefalakis, "Scalable and configurable end-to-end collection and analysis of IoT security data: towards end-to-end security in IoT systems," in 2019 Global IoT Summit (GIoTS), pp. 1-6, Aarhus, Denmark, 2019.

[47] W. Zhou, C. Cao, D. Huo et al., "Reviewing IoT security via logic bugs in IoT platforms and systems," IEEE Internet of Things Journal, vol. 8, no. 14, pp. 11621-11639, 2021.

[48] F. A. A. Lins and M. Vieira, "Security requirements and solutions for IoT gateways: a comprehensive study," IEEE Internet of Things Journal, vol. 8, no. 11, pp. 8667-8679, 2021.

[49] W. Wang, P. Xu, and L. T. Yang, "Secure data collection, storage and access in cloud-assisted IoT," IEEE Cloud Computing, vol. 5, no. 4, pp. 77-88, 2018.

[50] V. Hassija, V. Chamola, V. Saxena, D. Jain, P. Goyal, and B. Sikdar, "A survey on IoT security: application areas, security threats, and solution architectures," IEEE Access, vol. 7, pp. 82721-82743, 2019.

[51] S. Popli, R. K. Jha, and S. Jain, “A survey on energy efficient narrowband internet of things (NBIoT): architecture, application and challenges," IEEE Access, vol. 7, pp. 16739-16776, 2019.

[52] X. Ding and J. Wu, "Study on energy consumption optimization scheduling for internet of things," IEEE Access, vol. 7, pp. 70574-70583, 2019.

[53] H. M. Al-Kadhim and H. S. Al-Raweshidy, "Eergy efficient and reliable transport of data in cloud-based IoT," IEEE Access, vol. 7, pp. 64641-64650, 2019.

[54] C. Couso, J. Martin-Martinez, M. Porti, and M. Nafría, "Performance and power consumption trade-off in UTBB FDSOI inverters operated at NTV for IoT applications," IEEE Journal of the Electron Devices Society, vol. 6, pp. 55-62, 2018.

[55] S. Aljanabi and A. Chalechale, "Improving IoT services using a hybrid fog-cloud offloading," IEEE Access, vol. 9, pp. 1377513788, 2021. 